\section{SAT0482 SUBCLINICAL ENTHESOPATHY IN PSORIATIC PATIENTS AND ITS RELATION TO OTHER DISEASE PARAMETERS: AN ULTRASOUND STUDY}

W. Gouda ${ }^{1}$, M. Ismail ${ }^{2}$, M. Doma ${ }^{2} .{ }^{1}$ Rheumatology, Aswan University, Aswan; ${ }^{2}$ Rheumatology, Al-Azhar University, Assiut, Egypt

Background: Psoriasis is a chronic immune-mediated inflammatory skin disease characterized by reddish, thick patches covered with marked silvery scaling [1]. In about $70 \%$ of the patients, psoriasis is present many years before the onset of psoriatic arthritis [2]. The early recognition and therapeutic intervention especially with the new biologic treatments is critical to prevent the destructive and debilitating changes of psoriatic arthritis [3]. Enthesitis is inflammation at the attachment of tendons and ligaments to the bones, has been suggested as being the unifying feature of psoriatic arthritis, and the disease can be considered an enthesis associated disorder rather than primary synovitic arthropathy [4].

Objectives: We aimed to correlate the subclinical enthestitis in patients with psoriasis detected by means of power Doppler (PD) ultrasonography (US) with other disease parameters.

Methods: 50 persons with a definite diagnosis of psoriasis with no clinical evidence of arthritis or enthesitis were selected. All patients underwent complete clinical assessment included Psoriasis severity (PASI) score, body mass index (BMI), PDUS evaluation of Achilles, quadriceps, patellar entheses and plantar aponeurosis. US findings were scored according to the Glasgow Ultrasound Enthesitis Scoring System (GUESS).

Results: In 18 of 50 of patients (36\%) PDUS found signs indicative of enthesopathy. The Achilles enthesis had the highest number of PDUS signs of enthesopathy $(33.3 \%)$, followed by distal patellar enthesis $(22.2 \%)$, proximal patellar enthesis $(16.7 \%)$, quadriceps enthesis (16.7\%), and plantar aponeurosis enthesis $(11.1 \%$ ) with variable enthesial morphostructural abnormalities. The GUESS score was directly correlated with age $(p=0.012)$, disease duration $(p=0.044)$, PASI $(p=0.035)$, BMI $(p=0.011)$, hyperuricemia $(p=0.011)$.

Demographic findings of the study population

\begin{tabular}{lc}
\hline Characters & Patients \\
\hline Age (range) mean (SD) & $(19-70) 33.8 \pm 11.2$ \\
Gender & $31(62)$ \\
$\quad$ Female N (\%) & $19(38)$ \\
$\quad$ Male N (\%) & $(2-12) 7.7 \pm 3.4$ \\
Disease duration (range) mean (SD) & $21.1 \pm 7.3(2.7-33.2)$ \\
PASI score mean \pm SD (range) & $29.9 \pm 6.4(19.5-42)$ \\
BMI mean \pm SD (range) & \\
Rheumatoid factor: N of patients (\%) & $4(8 \%)$ \\
$\quad$ Positive (>1/8) & $46(92 \%)$ \\
$\quad$ Negative & $38(76 \%)$ \\
SUA: N of patients (\%) & $12(24 \%)$ \\
$\quad$ Normal (<6 mg/dl) & $6(12 \%)$ \\
$\quad$ Elevated & $32(64 \%)$ \\
Radiographic sacroiliitis: N of patients (\%) & $18(36 \%)$ \\
Ultrasonographic findings: (\%) & \\
$\quad$ Normal & \\
$\quad$ Abnormal (enthesitis) &
\end{tabular}

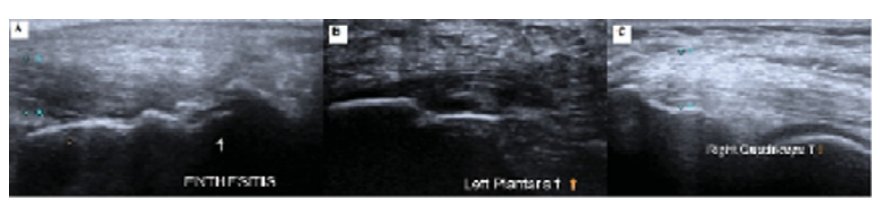

Conclusions: In addition to the importance of PDUS as a complimentery tool in examination of entheses in psoriatic patients, the presence of high PASI score together with the increased BMI and hyperuricemia in addition to long disease duration could be considered as predictive parameters for the presence of psoriatic enthesitis.

\section{References:}

[1] Menter A, Gottlieb A, Feldman SR, et al. Guidelines of care for the management of psoriasis and psoriatic arthritis, section 1. Overview of psoriasis and guide lines of care for the treatment of psoriasis with biologics. J Am Acad Dermatol. 2008; 58(5):826-50

[2] Dimitrios TB, loannis OT. Psoriatic arthritis. In: Klippel JH, ed.Primer on the Rheumatic Diseases. Atlanta, GA: Arthritis Foundation, 1997: 175-179.

[3] Rozenblit M, Lebwohl M. New biologics for psoriasis and psoriatic arthritis. Dermatol Ther. 2009; 22(1):56-60.

[4] McGonagle D, Conaghan PG, Emery P. Psoriatic arthritis: a unified concept twenty years on. Arthritis Rheum. 1999; 42:1080-7.

Disclosure of Interest: None declared

DOI: 10.1136/annrheumdis-2017-eular.1595
SATURDAY, 17 JUNE 2017 Osteoarthritis

\section{SAT0483 OSTEOARTHRITIS (OA) AND SOCIO-ECONOMIC STATUS (SES) PREDICTS THE ONSET OF COMORBIDITIES LINKED TO FREQUENT HEALTHCARE CONSULTATION}

R. Hayward ${ }^{1}$, K. Kaur ${ }^{2}$, R. Wilkie ${ }^{1} .{ }^{1}$ Research Institute for Primary Care \& Health Sciences; ${ }^{2}$ Medical Student, Keele University, Keele, United Kingdom

Background: $O A$ is the most common joint condition, the fourth leading cause of disability globally and the fastest increasing major health condition. In older adults, OA frequently coexists with other morbidities, but the temporal nature is unclear. There is abundant evidence that markers of low SES are associated with poor outcomes among people with musculoskeletal conditions, however the mechanism linking comorbidity may vary with SES.

Objectives: To examine if OA predicted the onset of comorbidities that are the reason for frequent consultation to primary care, and if this association was moderated by SES.

Methods: Cohort study combining questionnaire data at two time points (2005, 2008) in the North Staffordshire Osteoarthritis Project, and medical record data from 2000 to $2005(n=3910)$. OA was defined by consultation to primary care for OA between 2000 and 2005, and the indication of moderate to extreme pain interference in the questionnaire (2005). Logistic regression examined the association between $\mathrm{OA}$ and the onset of seven comorbidities (anxiety, depression, widespread pain (WP), insomnia, cognitive impairment, neurosis, stress between 2005 and 2008) and restricted social participation, first unadjusted and then adjusting for putative confounders (comorbidity, socio-demographic and lifestyle factors). Moderation of the association between OA and new onset comorbidity by change in income, education and area-level deprivation was examined by including interaction terms in regression and stratified analyses. Results were reported as odds ratios with a $95 \%$ Confidence Interval (OR; $95 \% \mathrm{Cl}$ ).

Results: Mean age was 63, 55\% were female, and 942 (24\%) had OA. In the unadjusted analysis, OA was significantly associated with new onset of seven comorbidities $(p<0.05)$. After adjusting for confounders, OA was associated with the onset of WP $(2.49 ; 1.96-3.17)$ and insomnia $(1.58 ; 1.14-2.19)$ There was a significant non-multiplicative interaction between $\mathrm{OA}$ and income and new onset cognitive impairment ( $P=0.047)$; new onset of cognitive impairment in those with OA whose income remains adequate $29.1 \%$ cf $38.1 \%$ in those with OA whose income remains inadequate), and between $O A$ and education and new onset WP ( $P=0.012$; new onset in those with $\mathrm{OA}$ and secondary education only was $37.4 \%$ cf $50 \%$ in those with $\mathrm{OA}$ and had more than a secondary education).

Conclusions: Consulters for OA were more likely to develop new physical and psychological comorbidities that lead to more frequent consultation to primary care than those without OA. Whilst confounders explained some of these associations, $\mathrm{OA}$ consulters may benefit from more proactive strategies to prevent further morbidity. Despite no significant multiplicative interactions, there were differences in the prevalence of new onset of morbidity in those with OA when stratified by SES. Onset of cognitive impairment was associated with inadequate income but WP was associated with those with higher education suggesting a "worried well" population seen both in other health surveys and in screening. OA and baseline morbidities were higher with lower SES and further exploration across the lifecourse will help to establish the role of SES on the natural history OA.

Disclosure of Interest: None declared

DOI: 10.1136/annrheumdis-2017-eular.2106

\section{SAT0484 LOW MAGNESIUM INTAKE IS ASSOCIATED WITH INCREASED PAIN IN SUBJECTS WITH RADIOGRAPHIC KNEE OSTEOARTHRITIS: DATA FROM THE OSTEOARTHRITIS} INITIATIVE

A. Shmagel ${ }^{1}$, N. Onizuka ${ }^{1}$, T. Vo ${ }^{2}$, L. Langsetmo ${ }^{2}$, K. Ensrud $^{3,4}$, R. Foley $^{5}$, P. Valen ${ }^{1,6}$. ${ }^{1}$ Medicine/Rheumatology; ${ }^{2}$ School of Public Health; ${ }^{3}$ Medicine, University of Minnesota; ${ }^{4}$ Medicine, VA Medical Center; ${ }^{5}$ Nephrology/Medicine, University of Minnesota; ${ }^{6}$ Medicine/Rheumatology, VA Medical Center, Minneapolis, United States

Background: Osteoarthritis pain appears to be caused, at least in part, by alterations in peripheral and/or central nociceptive pathways.[1] As magnesium is a known mediator of nociception, we hypothesized that low magnesium intake may be associated with increased pain in radiographic knee OA.

Objectives: To evaluate whether magnesium intake is associated with knee pain in radiographic knee osteoarthritis.

Methods: We investigated the associations between knee pain and magnesium intake from food and supplements in 2549 participants with prevalent radiographic knee OA (Kellgren-Lawrence score $\geq 2$ ) in the Osteoarthritis Initiative cohort.[2] WOMAC pain scores in the affected knee were reported annually with total follow up period of 48 months. Magnesium intake was assessed at baseline by food frequency questionnaire and dietary supplement questionnaire, and expressed in gender-specific calorie-adjusted quintiles. Analyses used generalized linear mixed effects models with repeated measures.

Results: Among participants with baseline radiographic knee OA the mean total magnesium intake was $310 \mathrm{mg} /$ day (SD 133) for men, and $288 \mathrm{mg} / \mathrm{day}$ (SD 